\title{
Neurosurgery in times of a pandemic: a survey of neurosurgical services during the COVID-19 outbreak in the Veneto region in Italy
}

\author{
Fabio Raneri, MD, ${ }^{1}$ Oriela Rustemi, MD,, Giampaolo Zambon, MD,, Giulia Del Moro, MD, ${ }^{2}$ \\ Salima Magrini, MD, ${ }^{3}$ Yuri Ceccaroni, MD, ${ }^{3}$ Elisabetta Basso, MD, ${ }^{4}$ Francesco Volpin, MD, ${ }^{5}$ \\ Martina Cappelletti, MD, ${ }^{2}$ Jacopo Lardani, MD, ${ }^{6}$ Stefano Ferraresi, MD, ${ }^{4}$ Franco Guida, MD, ${ }^{3}$ \\ Franco Chioffi, MD, ${ }^{5}$ Giampietro Pinna, MD, ${ }^{6}$ Giuseppe Canova, MD, ${ }^{2}$ Domenico d'Avella, MD, ${ }^{5,7}$ \\ Francesco Sala, MD, ${ }^{6}$ and Lorenzo Volpin, MD'
}

\begin{abstract}
${ }^{1}$ Azienda ULSS 8 Berica, Ospedale San Botolo, Vicenza; ${ }^{2}$ Azienda ULSS Marca Trevigiana, Ospedale Cà Foncello, Treviso; ${ }^{3}$ Azienda ULSS 3 Serenissima, Ospedale dell'Angelo, Mestre; ${ }^{4}$ Azienda ULSS 18 Rovigo, Ospedale Santa Maria della Misericordia, Rovigo; ${ }^{U}$ Università degli Studi di Padova, Azienda Ospedaliera di Padova, Padua; ${ }^{6}$ Università degli Studi di Verona, Azienda Ospedaliera Universitaria Integrata, Verona; and ${ }^{\top}$ Academic Neurosurgery, Department of Neurosciences, University of Padua Medical School, Padua, Italy
\end{abstract}

OBJECTIVE The purpose of this study was to analyze the effect of the coronavirus disease 2019 (COVID-19) outbreak and of the subsequent lockdown on the neurosurgical services of the Veneto region in Italy compared to the previous 4 years.

METHODS A survey was conducted in all 6 neurosurgical departments in the Veneto region to collect data about surgical, inpatient care and endovascular procedures during the month of March for each year from 2016 to 2020. Safety measures to avoid infection from SARS-CoV-2 and any COVID-19 cases reported among neurosurgical patients or staff members were considered.

RESULTS The mean number of neurosurgical admissions for the month of March over the 2016-2019 period was 663, whereas in March 2020 admissions decreased by 42\%. Emergency admissions decreased by 23\%. The average number of neurosurgical procedures was 697 , and declined by $30 \%$ (range $-10 \%$ to $-51 \%$ in individual centers). Emergency procedures decreased in the same period by $23 \%$. Subarachnoid hemorrhage and spontaneous intracerebral hemorrhage both decreased in Veneto-by $25 \%$ and $22 \%$, respectively. Coiling for unruptured aneurysm, coiling for ruptured aneurysm, and surgery for ruptured aneurysm or arteriovenous malformation diminished by $49 \%, 27 \%$, and $78 \%$, respectively. Endovascular procedures for acute ischemic stroke (AIS) increased by $33 \%$ in 2020 (28 procedures in total). There was a slight decrease (8\%) in brain tumor surgeries. Neurosurgical admissions decreased by $25 \%$ and $35 \%$ for head trauma and spinal trauma, respectively, while surgical procedures for head trauma diminished by $19 \%$ and procedures for spinal trauma declined by $26 \%$. Admissions and surgical treatments for degenerative spine were halved. Eleven healthcare workers and 8 patients were infected in the acute phase of the pandemic.

CONCLUSIONS This multicenter study describes the effects of a COVID-19 outbreak on neurosurgical activities in a vast region in Italy. Remodulation of neurosurgical activities has resulted in a significant reduction of elective and emergency surgeries compared to previous years. Most likely this is a combined result of cancellation of elective and postponable surgeries, increase of conservative management, increase in social restrictions, and in patients' fear of accessing hospitals. Curiously, only endovascular procedures for AIS have increased, possibly due to reduced physical activity or increased thrombosis in SARS-CoV-2. The confounding effect of thrombectomy increase over time cannot be excluded. No conclusion can be drawn on AIS incidence. Active monitoring with nasopharyngeal swabs, wearing face masks, and using separate pathways for infected patients reduce the risk of infection.

https://thejns.org/doi/abs/10.3171/2020.9.FOCUS20691

KEYWORDS neurosurgery; COVID-19; SARS-CoV-2; emergency; surgery; pandemic

ABBREVIATIONS AIS = acute ischemic stroke; $\mathrm{HDU}=$ high-dependency unit; ICU = intensive care unit; OR = operating room; SAH = subarachnoid hemorrhage SUBMITTED July 31, 2020. ACCEPTED September 21, 2020.

INCLUDE WHEN CITING DOI: 10.3171/2020.9.FOCUS20691. 
$\mathrm{T}$ HE recent outbreak of severe acute respiratory syndrome coronavirus 2 (SARS-CoV-2)-related disease (i.e., coronavirus disease 2019 or COVID-19) spread to pandemic proportions and increased demands and pressure on health systems all over the world. Italy became the epicenter of the epidemic in March 2020, with a toll of 110,574 infected, 13,155 deaths, and 4035 patients occupying intensive care units (ICUs) on April 1, 2020. As the public health emergency escalated, hospitals transformed operating rooms (ORs) and reallocated anesthesia staff to increase ICU capacity. Elective surgery was brought to a standstill, clinics were cancelled, and medical staff began training for noninvasive ventilation. Neurosurgical departments were forced to put off elective and postponable surgeries, guaranteeing only emergency or high-priority operations. A national lockdown was ordered on March 9 to control the pressure on hospitals and to reduce the increasing rate of infection. We analyze the effect of the COVID-19 outbreak and of the lockdown on the neurosurgical services of the Veneto region in Italy compared to the previous 4 years, as well as the safety measures adopted by institutions and neurosurgical departments. We also describe COVID-19 cases reported among neurosurgical patients or staff members.

\section{Methods}

A survey was conducted in all the neurosurgical departments in the Veneto region in Italy to collect data about surgical and inpatient care during the month of March for each year from 2016 to 2020. Data collected were number of admissions, emergency/urgent admissions, overall number of surgical procedures, and emergency/ urgent operations. We further considered endovascular embolization for aneurysms (ruptured or unruptured), endovascular revascularization for stroke, procedures for degenerative spine, operations for head and spinal trauma, nontraumatic hemorrhage, brain tumor, and pediatric procedures. Data were extracted from hospital registries. We further considered the number of beds on neurosurgical wards, neuro-ICUs, and our high-dependency units (HDUs) (when present), and also the number of operative sessions (considered as 6-hour sessions). Each variable considered was analyzed by individual institution and by overall regional volume. We compared available data of the month of March 2020 with the average of the same month in the 4-year period between 2016 and 2019. It was not the purpose of our study to discuss the strength of the individual departments.

We also considered regional and single institutions' guidelines for patients' and healthcare workers' safety to avoid infection from SARS-CoV-2, and any COVID-19 cases reported among neurosurgical patients or staff members.

Statistical analysis was performed to obtain percentages and mean values. Regional data were collected by considering the sum of means of single institutions for the 4-year period from 2016 to 2019 and the sum of single-institution results for the 2020 period (QuickCalcs [GraphPad Software, Inc; Statcon] and Microsoft Excel 2011, version 14.3.6).

\section{Results}

All 6 neurosurgical departments in the Veneto region completed the survey (Table 1). Two institutions are academic centers running a residency program, and the remaining 4 are tertiary teaching hospitals for neurosurgery. General characteristics of variations in patient admissions, procedures, operative sessions, and beds on wards and in the neuro-ICU are summarized in Table 2. Both academic hospitals and 1 tertiary hospital report a dedicated neuroICU with a total of 34 beds in the region. Three centers report a general ICU without dedicated beds for neurosurgery or neurotrauma. One center usually has $12 \mathrm{HDU}$ beds on the ward.

During March 2020, 2 institutions reported a decline of dedicated neuro-ICU beds due to COVID-19 reorganization, with a total loss of 10 beds $(-29 \%)$. One academic center reported no variation of neuro-ICU beds. One center increased HDU beds (from 0 to 4); the same center had lost 4 dedicated ICU beds.

The mean neurosurgical admissions in the Veneto region over the 4-year period between 2016 and 2019 had been 663 during the month of March, while in the same time span in 2020 the total admissions decreased to 386 $(-42 \%)$. Only 1 institution reported a minor reduction in neurosurgical admissions $(-17 \%)$, whereas 5 departments claimed reductions of $40 \%$ to more than $50 \%$ (Fig. 1). Emergency admissions decreased by $23 \%$ in the region (range $-12 \%$ to $-43 \%$ in individual centers).

The average number of neurosurgical procedures in the entire region between 2016 and 2019 in the whole month of March was 697 operations (range 60-193 per institution). The overall amount of neurosurgical procedures declined to $486(-30 \%$, range $-10 \%$ to $-51 \%$ in individual centers). Emergency procedures decreased in the same period by 23\% (from an average of 216 between 2016 and 2019 to 167 in 2020). Two institutions did not record a significant variation in emergency operations compared to the previous 4 years.

Five neurosurgical departments had suffered reductions in elective operative sessions ( $-7 \%$ to $-75 \%$; overall loss of 94 sessions in Veneto). One neurosurgical department reported a minor change in routine surgical practice with cancellation of pediatric operative sessions.

Admissions and procedures according to clinical conditions are summarized in Figs. 2 and 3. Subarachnoid hemorrhage (SAH) and spontaneous intracerebral hemorrhage (without aneurysm rupture or trauma) both decreased in Veneto-by 25\% and 22\%, respectively. Coiling for unruptured aneurysm, coiling for ruptured aneurysm, and surgery for aneurysm or arteriovenous malformation bleeding diminished by $49 \%, 27 \%$, and $78 \%$, respectively, in the whole region. Curiously, in the same period endovascular procedures for acute ischemic stroke (AIS) increased by $33 \%$ in 2020, with 28 procedures being performed.

One hundred twenty-three brain tumors were operated on in the whole region in March 2020 against an average of 134 tumors per year in the previous 4 years $(8 \%$ less). Two centers reported a reduction of more than $30 \%$ in brain tumor surgery, whereas the other 4 institutions reported minor variations. 
TABLE 1. Public institutions in Veneto invited to participate in the survey on neurosurgical activities during the COVID-19 pandemic
1. Azienda ULSS 3 Serenissima, Ospedale dell'Angelo, Mestre
2. Università degli Studi di Padova, Azienda Ospedaliera di Padova, Padua
3. Azienda ULSS 18 Rovigo, Ospedale Santa Maria della Misericordia, Rovigo
4. Azienda ULSS Marca Trevigiana, Ospedale Cà Foncello, Treviso
5. Università degli Studi di Verona, Azienda Ospedaliera Universitaria Integrata, Verona
6. Azienda ULSS 8 Berica, Ospedale San Botolo, Vicenza

Neurosurgical admissions decreased by $25 \%$ and $35 \%$ for head trauma and spinal trauma, respectively, during March 2020 in the entire region, while surgical procedures for head trauma diminished by $19 \%$ and procedures for spinal trauma declined by $26 \%$. Admissions and operations for degenerative spine cases were halved in the same period. Nine pediatric procedures were performed in March 2020 - mostly in 2 hub institutions (D and E) against a mean of 19 in the previous 4 years (50\% less).

Local institutions and regional guidelines required a negative nasopharyngeal swab for SARS-CoV-2 before admission for patients undergoing elective procedures (within 48 hours). Elective patients could only receive surgery if they had a negative swab result, which had to be repeated if more than 6 days had elapsed at the time of surgery. All medical, nursing, and other healthcare professionals wore surgical masks at all times, sanitized their hands frequently, and wore disposable gloves. Patients admitted on an urgent or emergency basis were kept in a separate "filter" room that was only accessed if necessary and with adequate protective gear until the swab result was available. Any patients positive for SARS-CoV-2 were immediately transferred to COVID-19-dedicated wards and managed by dedicated staff. Family and visitors were not allowed in the wards and any information was given only by phone or conference calls. All meetings were cancelled or performed in conference calls. Staff were actively monitored with a nasopharyngeal swab every 7 days if contact with patients affected by COVID-19 occurred. Otherwise, swabs were performed every 20 days. Any positive staff members were put in isolation at home or admitted to
COVID wards if necessary. SARS-CoV-2 cases in neurosurgical departments are described in Table 3. Only 1 healthcare worker needed hospital care (with intubation and intensive care).

\section{Discussion}

As the COVID-19 pandemic advanced, Fontanella et al. showed through a worldwide survey how surgeries were reduced globally by more than $70 \%,{ }^{1}$ with surgeons performing only unpostponable procedures such as resection of tumors with mass effect or emergency procedures (in some cases entire neurosurgical departments were closed or surgeries were centralized to high-volume centers). No changes in regard to SAH management were reported. ${ }^{1}$ Indications given by international societies confirmed the value of delaying elective procedures to contain the spread of SARS-CoV-2, to reduce the patient load in ICUs with non-COVID-19 patients, and to reduce the possibility of treating asymptomatic patients with SARS-CoV-2 who would be at high risk of deteriorating and would increase the risk of infecting health professionals and other patients. ${ }^{1-6}$

Italy became the epicenter of the epidemic in March 2020 , with a toll of 110,574 infected, 13,155 deaths, and 4035 patients occupying ICUs on April 1, 2020. These numbers were concentrated mostly in the northern regions of Veneto, Piedmont, Emilia-Romagna and, most of all, Lombardy. Hospitals transformed ORs and reallocated anesthesia staff to increase ICU capacity.

Several criteria for identifying and for time-managing

TABLE 2. Variations in general activity and emergency activities in individual centers and the mean variations in activities in the Veneto region

\begin{tabular}{|c|c|c|c|c|c|c|c|c|}
\hline \multirow[b]{2}{*}{ Institutions } & \multicolumn{8}{|c|}{ No. (\%) Change } \\
\hline & $\begin{array}{c}\text { Total } \\
\text { Admissions }\end{array}$ & $\begin{array}{l}\text { Emergency } \\
\text { Admissions }\end{array}$ & Ops & $\begin{array}{c}\text { Emergency } \\
\text { Ops }\end{array}$ & $\begin{array}{c}\text { 6-Hr Surgical } \\
\text { Sessions }\end{array}$ & $\begin{array}{l}\text { Hospital } \\
\text { Beds }\end{array}$ & $\begin{array}{c}\text { No. of } \\
\text { HDU Beds }\end{array}$ & $\begin{array}{c}\text { No. of } \\
\text { Neuro-ICU Beds }\end{array}$ \\
\hline$A$ & $-50(-41 \%)$ & $-5(-12 \%)$ & $-57(-46 \%)$ & $-16(-33 \%)$ & $-19(-43 \%)$ & NV & NV & NA \\
\hline$B$ & $-29(-45 \%)$ & $-3(-12 \%)$ & $-28(-47 \%)$ & $-10(-36 \%)$ & $-13(-48 \%)$ & $-4(-20 \%)$ & NA & NA \\
\hline C & $-29(-40 \%)$ & $-5(-26 \%)$ & $-33(-51 \%)$ & +1 (+5\%) & $-25(-75 \%)$ & $-7(-47 \%)$ & NA & NA \\
\hline$D$ & $-13(-17 \%)$ & $-9(-23 \%)$ & $-7(-10 \%)$ & $-3(-20 \%)$ & $-5(-7 \%)$ & $0^{*}$ & NA & $0(0 \%)$ \\
\hline$E$ & $-103(-51 \%)$ & $-10(-20 \%)$ & $-43(-22 \%)$ & $+1(+3 \%)$ & $-16(-18 \%)$ & $-6(-9 \%)$ & NA & $-6(-32 \%)$ \\
\hline $\mathrm{F}$ & $-54(-41 \%)$ & $-18(-43 \%)$ & $-44(-23 \%)$ & $-22(-31 \%)$ & $-17(-25 \%)$ & $-12(-43 \%)$ & NA & $-4(-50 \%)$ \\
\hline $\begin{array}{l}\text { Total, } \\
\text { Veneto }\end{array}$ & $-277(-42 \%)$ & $-49(-23 \%)$ & $-211(-30 \%)$ & $-49(-23 \%)$ & $-94(-48 \%)$ & $-40(-20 \%)$ & $+4(+29 \%)$ & $-10(-29 \%)$ \\
\hline Mean & $-46(-39 \%)$ & $-8(-23 \%)$ & $-35(-33 \%)$ & $-8(-19 \%)$ & $-16(-36 \%)$ & $-5(-24 \%)$ & & \\
\hline
\end{tabular}

$\mathrm{NA}=$ none available; $\mathrm{NV}=$ no variation.

* Eleven-bed reduction due to renovations already in place at the time of the COVID-19 outbreak-these data were not considered. 


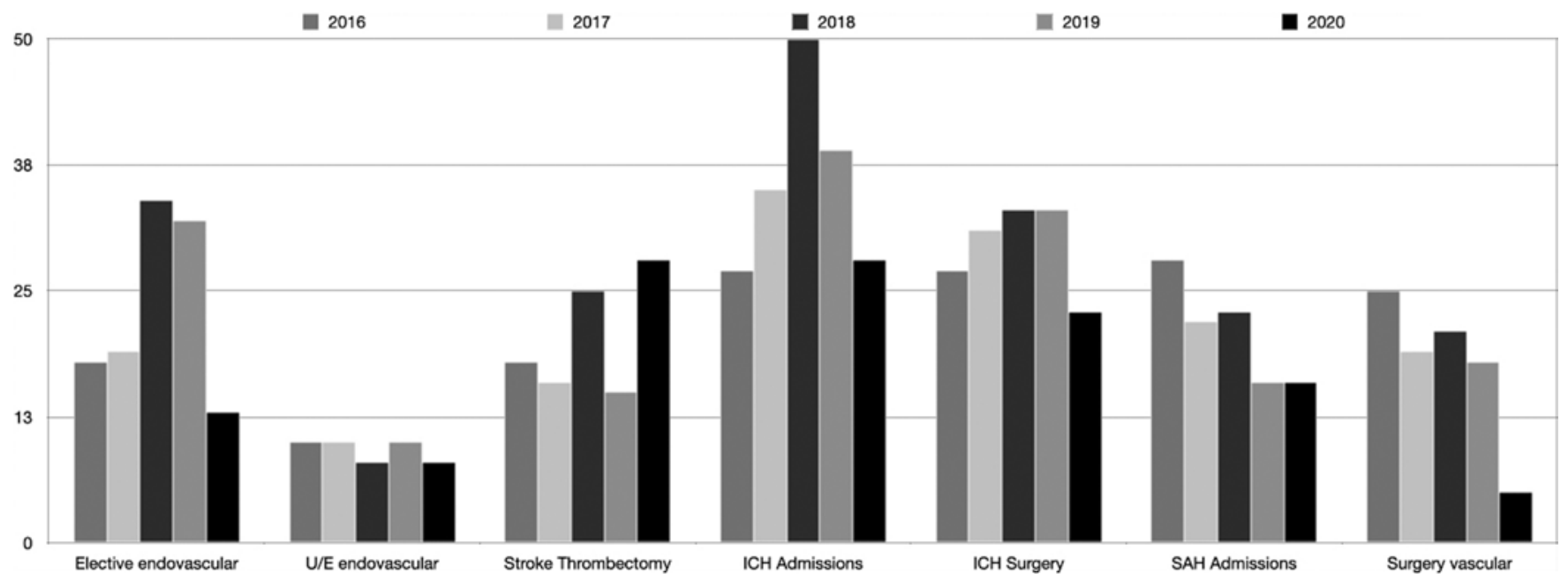

FIG. 1. Overall amount of elective endovascular procedures, urgent/emergency (U/E) endovascular procedures, endovascular stroke endothrombectomy, admissions for intracerebral hemorrhage $(\mathrm{ICH})$ and $\mathrm{SAH}$, total number of surgeries for intracerebral hemorrhage, or vascular surgery performed during the month of March in all neurosurgical departments in the Veneto region in Italy are shown for each year from 2016 to 2020 . The increments on the $y$-axis denote the number of admissions or procedures performed.

nonpostponable neurosurgical procedures were attempted. ${ }^{7,8}$ Further indications regarded spine surgery.,10 Burke et al. ${ }^{11}$ presented a 3 -tier system for surgery scheduling considering the number of community cases, COVID-19positive inpatients, and percentage of staffing shortages. A checklist was created to determine the case urgency, availability of ORs, and postoperative beds. ${ }^{11}$ Schiariti et al. ${ }^{12}$ underlined the need for predictors that may help in the decision-making process such as machine learning on Big Data. They suggested that medical registries supported by individual nations and the WHO be encouraged to collect factors enabling the building of predictive models useful for the future. ${ }^{12}$ Strong indications were given to avoid transsphenoidal approaches for pituitary tumors due to the extremely high transmission risk and to favor open craniotomy for deteriorating visual deficits, as well as pituitary apoplexy causing rapid loss of vision. ${ }^{13,14}$

Our experience concentrates on the Veneto region, which counts an overall population of 4.9 million and a territory of $18,345.35 \mathrm{~km}^{2}$ (population density 267.59 inhabitants $/ \mathrm{km}^{2}$ ), resulting in the second most densely populated region in Italy. The National Health Service is independently programmed by the regional governments and is predominantly public in Veneto. There are 6 neurosurgical departments with approximately 75 neurosurgeons organized on a geographical rather than population-based division. The first 2 cases in the region were reported on February 20, and the first death was on February 21 (the

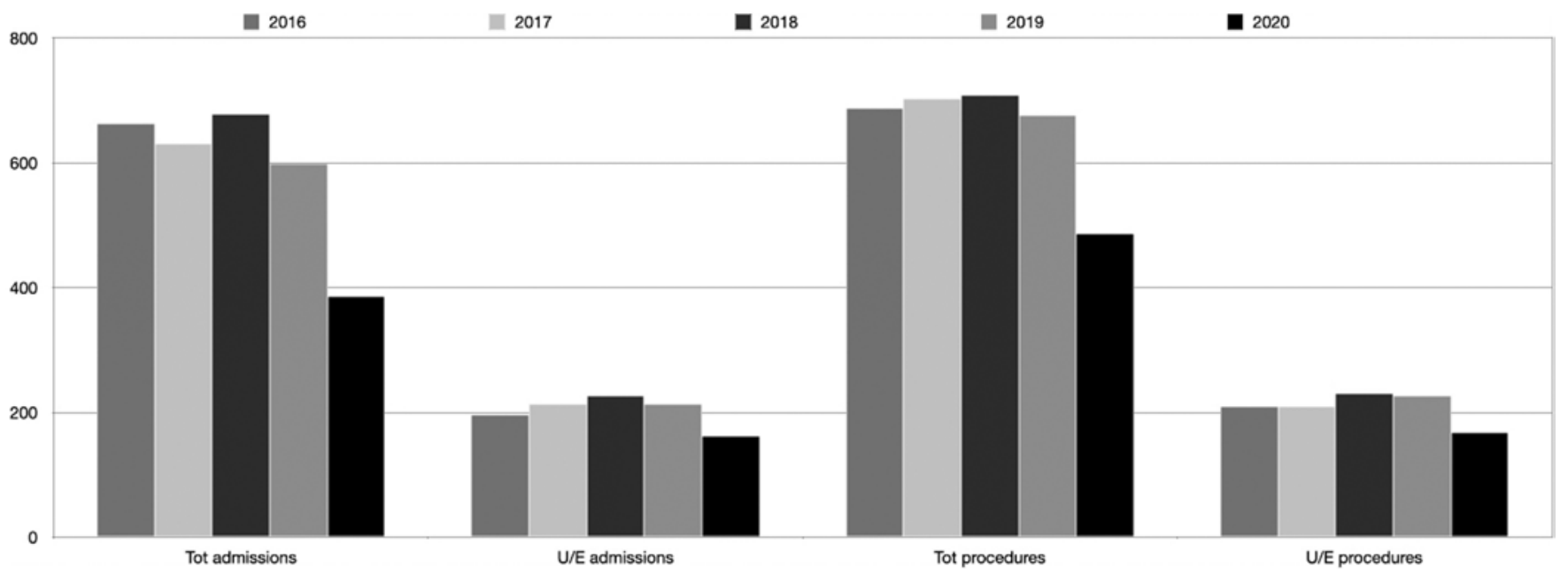

FIG. 2. Total (Tot) global admissions, urgent/emergency (U/E) admissions, total amount of procedures, and total amount of urgent/ emergency procedures performed during the month of March in all neurosurgical departments in the Veneto region in Italy are shown for each year from 2016 to 2020. The increments on the $y$-axis denote the number of admissions or procedures performed. 


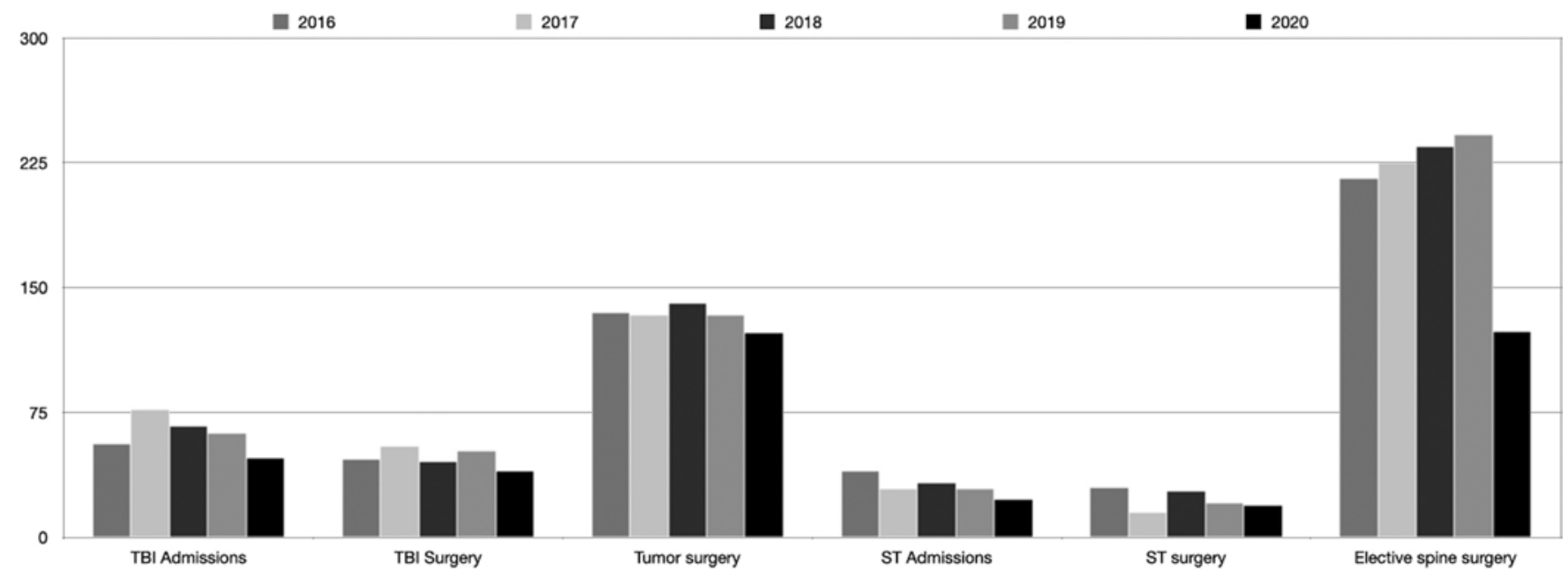

FIG. 3. Overall number of admissions for traumatic brain injury (TBI) and spinal trauma (ST), surgical procedures for TBIs and ST, surgeries for intracranial tumors, and elective spine procedures performed during the month of March in all neurosurgical departments in the Veneto region in Italy are shown for each year from 2016 to 2020. The increments on the y-axis denote the number of admissions or procedures performed.

first death due to COVID-19 in Italy). By April 1, 2020, the Veneto region counted 9625 infected, 499 deaths, and 350 patients in ICUs. An extensive SARS-CoV-2 molecular testing policy was adopted..$^{15}$

During the month of March elective surgeries were brought to a standstill, clinics were cancelled, and medical staff began training for noninvasive ventilation. Neurosurgical departments were forced to put off elective and postponable surgeries, guaranteeing only emergency or high-priority operations (such as brain tumors). A national lockdown was ordered on March 9 to control the pressure on hospitals and to reduce the increasing rate of infection. Health authorities requested hospitals to limit OR activities to emergencies, urgencies, and oncological cases. Neurosurgical activities slowed down, adapting to the crisis and to the limits of usual resources (in particular ORs and ICU beds). Emergency and urgent operations for trauma, stroke, $\mathrm{SAH}$, acute hydrocephalus, head trauma, and spine trauma were guaranteed. Operations for tumors at risk of giving rise to intracranial hypertension, malignant tumors, and spinal cord compressions with or at risk of neurological deficits were scheduled according to availability of ORs and ICU beds (class A patients). Patients were tested within 48 hours of hospital admission for SARS-CoV-2 virus. Emergency procedures in patients without a swab result were performed in dedicated COVID-19 ORs with negative pressure and necessary personal protective equipment.

All neurosurgical departments remained active in Veneto during the pandemic. Neurosurgeons were not allocated outside their departments; the reorganization was only intradepartmental. Conversely, in Lombardy the neurosurgical activity was reorganized in 4 hub hospitals, leaving spoke hospitals inactive. . $^{16,17}$ This probably reflected the heavier COVID-19 burden in that region.

We believe this could be due to multiple factors differentiating the demographic factors and healthcare programs of the 2 regions, although it will take some time to adequately analyze. First, the Veneto region isolated their initial foci immediately and rapidly started a swab campaign to identify, track, and treat both symptomatic and asymptomatic patients or healthcare workers to avoid vast and uncontrolled diffusion of infection. Lombardy reported 2 main foci at the epidemic outbreak, although only 1 was adequately isolated and quarantined while much time was lost before measures were taken for the second outbreak in the area of Bergamo, sadly leading to one of the worst-hit areas in the world.

Second, the Veneto region presents a more rural demographic composition with a lower population density than Lombardy (270 inhabitants per $\mathrm{km}^{2}$ vs 420 per km², respectively), whereas Lombardy is dominated by the metropolitan area of Milan and its financial and commercial importance. In fact, the hardest-hit areas were large cities and metropolitan areas such as Madrid, Paris, London, and New York rather than smaller cities.

Furthermore, whereas in Lombardy even mildly symp-

TABLE 3. Healthcare workers testing positive for SARS-CoV-2 on nasopharyngeal swab or affected by COVID-19 in neurosurgical departments of individual institutions in the Veneto region in March 2020

\begin{tabular}{cccccc}
\hline Institutions & $\begin{array}{c}\text { Medical } \\
\text { Doctors }\end{array}$ & Nurses & $\begin{array}{c}\text { Other Healthcare } \\
\text { Workers }\end{array}$ & Patients & Total \\
\hline A & 0 & 0 & 2 & 3 & 5 \\
\hline B & 0 & 0 & 0 & 0 & 0 \\
\hline C & 0 & 0 & 0 & 1 & 1 \\
\hline D & 2 & 0 & 0 & 0 & 2 \\
\hline E & 0 & 0 & 0 & 0 & 0 \\
\hline F & 3 & 4 & 0 & 4 & 11 \\
\hline Total & 5 & 4 & 2 & 8 & 19 \\
\hline
\end{tabular}

All medical and nursing staff were actively monitored with serial nasopharyngeal swabs. 
tomatic patients were hospitalized due to a less expansive territorial medicine program, rapidly filling available beds, in Veneto asymptomatic patients or those with mild symptoms were kept home in isolation and were actively monitored with serial swab tests.

Last, the health system in Veneto is mostly public, with a few minor private hospitals, whereas Lombardy has a competitive public and private system with approximately one-third of overall beds available in the private/semiprivate hospitals. This factor probably delayed coordination for rapid and homogeneous action to contain infection in Lombardy, whereas in Veneto separate pathways and dedicated COVID-19 ICUs were created overnight and protective measures were enforced immediately.

In this paper we analyzed the effect of the COVID-19 outbreak on the neurosurgical services of the Veneto region in Italy compared to the previous 4 years. Overall, there are 6 neurosurgical departments in the region performing an average of 697 neurosurgical procedures in the month of March (average data of the month of March from 2016 to 2019). Of these, 216 are emergency/urgent procedures. After the first month from the outbreak of the COVID-19 pandemic 486 surgeries had been performed, with 167 being urgent/emergency procedures $(-30 \%$ and $-23 \%$, respectively). Among all 6 institutions only 1 experienced a mild reduction in surgical activity (hospital D, $10 \%$ reduction), whereas 3 showed a significant decrease in surgical procedures ranging between $46 \%$ and $51 \%$ reductions (hospitals A, B, and C). The remaining 2 institutions, hospitals $\mathrm{E}$ and $\mathrm{F}$, showed moderate reductions of the number of surgical procedures (22\% and $23 \%)$. Curiously, the institutions reporting milder reductions all have a dedicated neuro-ICU and 2 are academic centers, whereas the institutions reporting an approximately $50 \%$ reduction have a general ICU. These data appear to confirm previous considerations that departments with greater resources (such as a dedicated ICU, higher number of operative sessions, or more extensive teaching facilities) seem to be better protected from disruption of usual activities.

We experienced a significant decrease in overall neurosurgical admissions, mostly due to reduction of elective cases and emergency admissions, probably supported by an increase in conservative management, patients' fear of going to hospitals seen as a risk of infection, and a reduction of trauma cases due to the national lockdown measures..$^{18-20}$

The most interesting data from the analysis of our region's neurosurgical departments' activities during March 2020 was the increase (33\%) of endovascular procedures for AIS, whereas globally admissions and surgeries/endovascular procedures for hemorrhagic stroke and SAH decreased considerably. Although isolated cases of aneurysmal SAH in SARS-CoV-2-positive patients have been reported ${ }^{21}$ a global reduction of SAH caused by aneurysm rupture was noticed by other authors. ${ }^{22,23}$ We believe that the increase in endovascular procedures for ischemic stroke may be due to the sedentary lifestyle during social lockdown, although it may also be due to prothrombotic characteristics of COVID-19. In this survey we did not investigate the possible portion of patients with SARS$\mathrm{CoV}-2$ infection. Recent randomized controlled trials have extended the indications of endovascular thrombectomy; however, overall pooled data collected should mitigate that effect. A better investigation of this finding would require a dedicated study considering also if there was a change in AIS admissions and systemic thrombolysis. Data from another study showed stable thrombectomy numbers but a 30\% AIS reduction in emergency departments. ${ }^{24}$ A 39\% reduction of patients receiving radiological evaluation for AIS emerged in a large North American analysis. ${ }^{25} \mathrm{~A}$ multicenter retrospective observational study collecting data from different countries showed a $32 \%$ reduction of endovascular procedures for AIS. ${ }^{26}$ Data from a tertiary referral center indicated a significant decline in AIS admissions by $23 \%$; in contrast, mechanical thrombectomy procedures increased by $50 \%$, with $25 \%$ of thrombectomy patients positive for COVID-19. ${ }^{27}$ Caution must be taken in data interpretation. None of the studies has the proper design to answer questions on effective AIS incidence during the pandemic. Real incidence must not be confused with a decline of patients seeking medical care.

Few studies to our knowledge have so far analyzed the actual variation of neurosurgical procedures in a multicenter survey covering a vast region at the time of greatest restrictions in practice due to a COVID-19 outbreak. ${ }^{23,28}$ Mazzatenta et al. reported a decrease of $29.6 \%$ for emergency/urgent procedures and $21.6 \%$ for tumors. ${ }^{23}$ However, that study is limited by differing periods considered by each institution, and the analysis compared similar periods of 2020 with 2018 and 2019.

Few cases among healthcare workers were reported during the period considered and no cases were reported in the following 2 months. All cases were reported in the earlier part of the month and were due to unpreparedness and unawareness at epidemic outbreak as already described by Rustemi et al. ${ }^{2}$ These data mean that adequate protective gear in a COVID-19-free ward can significantly reduce exposure and risk of infection if adequate safety measures are adopted (described in Table 4).

Our study does not consider outpatient clinics' reductions because clinics were cancelled to limit circulation of people in hospitals and to avoid crowded waiting rooms. Only urgent patients had access to clinics. Ward visits were also suspended. Moreover, although certain procedures such as trauma surgery $(-25 \%)$ have diminished, probably due to measures enforced by the national lockdown, reductions of surgeries for tumors $(-8 \%)$ and reductions of admissions for SAH $(-29 \%)$ or for hemorrhagic stroke $(-28 \%)$ cannot be directly correlated to the same measures. We fear that the missing numbers are filling a Pandora's box with patients waiting for an operation that will be opened in the following months when neurosurgical practices will try to recover. Our next challenge will be how to face a sudden increase of patients needing an operation in uncertain times characterized by numerous foci of COVID-19. Guidelines should be prepared regionally according to resources and to the epidemiological impact of COVID-19 in a multi-tier fashion to avoid finding ourselves unprepared to handle resumption of normal hospital functions as well as possibly rewinding back to a department lockdown as foci may blossom. ${ }^{29}$ It is our duty to keep alive the memory of the hard times faced over the last months to protect our patients and ourselves from the next pandemic. 
TABLE 4. Preparedness for the next pandemic from airborne pathogens or surge of COVID-19

\begin{aligned} & \hline 1. Face masks. \\ & \hline 2. Hand washing. \\ & \hline 3. Nasopharyngeal swabs to monitor staff and patients. \\ & \hline 4. Separate pathways and wards for infected patients (both symptomatic and asymptomatic). \\ & \hline 5. Isolate healthcare workers with a positive nasopharyngeal swab. \\ & \hline 6. Isolate all patients until swab result available (filter room). \\ & \hline 7. Avoid unecessary contact with patients waiting for a swab result. \\ & \hline 8. Close department to visitors and meetings. \\ & \hline 9. Use telemedicine and conference calls for meetings or consultations. \\ & \hline 10. Postpone nonurgent and elective procedures. \\ & \hline 11. Possibly use dedicated hospitals and personnel (including radiology, ICU, infectious disease, \\ & pneumonology, laboratory medicine, and microbiology specialists). \\ & \hline\end{aligned}

\section{Conclusions}

This multicenter study describes the consequences of a COVID-19 outbreak on neurosurgical activities in a vast region in Italy at the time of the maximum pressure thus far on the public health system. Remodulation of neurosurgical activities has resulted in significant reductions of elective surgery and emergency procedures compared to previous years. Most likely this is a combined result of cancellation of elective and postponable surgeries, increase of conservative management, social restrictions, and patients' fear of accessing hospitals. Curiously, only endovascular procedures for AIS have increased in this period, possibly due to reduced physical activity during social lockdown or possibly to increased thrombosis in SARS-CoV-2 infections. No conclusion can be drawn on the incidence of AIS during this pandemic.

Monitoring healthcare workers as well as patients with nasopharyngeal swabs, wearing masks, and separating infected patients in dedicated wards appears to considerably reduce the risk of infection and should be enforced for any airborne pathogens in the next pandemic.

\section{References}

1. Fontanella MM, De Maria L, Zanin L, et al. Neurosurgical practice during the severe acute respiratory syndrome coronavirus 2 (SARS-CoV-2) pandemic: a worldwide survey. World Neurosurg. 2020;139:e818-e826.

2. Rustemi O, Segna A, Zanusso M, et al. Covid-19 diffusion in a neurosurgical "clean" department: the asymptomatic Trojan horse. Acta Neurochir (Wien). 2020;162(7):1507-1508.

3. COVID-19 in neurosurgery. News, guidelines and discussion forum. European Association of Neurosurgical Societies. Accessed October 15, 2020. https://www.eans.org/page/covid-19

4. Current emergencies. CMS.gov. Accessed October 15, 2020. https://www.cms.gov/About-CMS/Agency-Information/ Emergency/EPRO/Current-Emergencies/Current-Emergenciespage

5. American College of Surgeons. COVID-19: Recommendations for management of elective surgical procedures. March 13, 2020. Accessed October 15, 2020. https://www.facs.org/ covid-19/clinical-guidance/elective-surgery

6. Lee ZD, Chyi Yeu DL, Ang BT, et al. Editorial. COVID-19 and its impact on neurosurgery: our early experience in Singapore. J Neurosurg. 2020;133(1):24-25.

7. Zoia C, Bongetta D, Veiceschi P, et al. Neurosurgery during the COVID-19 pandemic: update from Lombardy, northern Italy. Acta Neurochir (Wien). 2020;162(6):1221-1222.

8. Agosti E, Giorgianni A, Pradella R, Locatelli D. Coronavirus disease 2019 (COVID-19) outbreak: single-center experience in neurosurgical and neuroradiologic emergency network tailoring. World Neurosurg. 2020;138:548-550.

9. Donnally CJ III, Shenoy K, Vaccaro AR, et al. Triaging spine surgery in the COVID-19 era. Clin Spine Surg. 2020;33(4): $129-130$.

10. Zou J, Yu H, Song D, et al. Advice on standardized diagnosis and treatment for spinal diseases during the coronavirus disease 2019 pandemic. Asian Spine J. 2020;14(2):258-263.

11. Burke JF, Chan AK, Mummaneni V, et al. In reply: The coronavirus disease 2019 global pandemic: a neurosurgical treatment algorithm. Neurosurgery. 2020;87(3):E407.

12. Schiariti M, Restelli F, Broggi M, et al. In reply: The coronavirus disease 2019 global pandemic: a neurosurgical treatment algorithm. Neurosurgery. 2020;87(2):E216-E217.

13. Mitchell RA, King JAJ, Goldschlager T, Wang YY. Impact of COVID-19 on pituitary surgery. ANZ J Surg. 2020;90(6): 963-964.

14. Castelnuovo P, Turri-Zanoni M, Karligkiotis A, et al. Skullbase surgery during the COVID-19 pandemic: the Italian Skull Base Society recommendations. Int Forum Allergy Rhinol. 2020;10(8):963-967.

15. Lavezzo E, Franchin E, Ciavarella C, et al. Suppression of a SARS-CoV-2 outbreak in the Italian municipality of Vo'. Nature. 2020;584(7821):425-429.

16. Giussani C, Sganzerla E, Valvassori L, et al. The response during a pandemic is a blurred vision of the future. Reflections on the Lombardy reorganization of the neurosurgical emergencies during the COVID-19. Acta Neurochir (Wien). 2020;162(6):1225-1226.

17. Cellerini M, Valvassori L, Ruggiero M, et al. Redesigning Italy's regional neurosciences networks in the COVID-19 era: a model for a future healthcare system or skating on thin ice? J Neurosurg Sci. Published online June 17, 2020. doi:10.23736/S0390-5616.20.05019-5

18. Dobran M, Paracino R, Iacoangeli M. Letter to the editor by Dobran Mauro, Paracino Riccardo, and Iacoangeli Maurizio regarding "Neurosurgery during the COVID-19 pandemic: update from Lombardy, northern Italy." Zoia C, Bongetta D, Veiceschi P, Cenzato M, Di Meco F, Locatelli D, Boeris D, Fontanella MM. Acta Neurochir (Wien). 2020 Mar 28. doi: 10.1007/s00701-020-04305-w. Acta Neurochir (Wien). 2020; 162(6):1223-1224.

19. Borsa S, Pluderi M, Carrabba G, et al. Letter to the editor: Impact of COVID-19 outbreak on acute low back pain. World Neurosurg. 2020;139:749. 
20. Cenzato M, DiMeco F, Fontanella M, et al. Editorial. Neurosurgery in the storm of COVID-19: suggestions from the Lombardy region, Italy (ex malo bonum). J Neurosurg. 2020;133(1):33-34.

21. Rustemi O, Raneri F, Iannucci G, et al. Aneurysmal subarachnoid hemorrhage in a SARS-CoV-2 positive testing: casual or causal? Letter. Br J Neurosurg. Published online June 22, 2020. doi:10.1080/02688697.2020.1787343

22. Bernat AL, Giammattei L, Abbritti R, Froelich S. Impact of COVID-19 pandemic on subarachnoid hemorrhage. J Neurosurg Sci. 2020;64(4):409-410.

23. Mazzatenta D, Zoli M, Cavallo MA, et al. Remodulation of neurosurgical activities in an Italian region (Emilia-Romagna) under COVID- 19 emergency: maintaining the standard of care during the crisis. J Neurosurg Sci. Published online June 11, 2020. doi:10.23736/S0390-5616.20.05018-3

24. Uchino K, Kolikonda MK, Brown D, et al. Decline in stroke presentations during COVID-19 surge. Stroke. 2020;51(8): 2544-2547.

25. Kansagra AP, Goyal MS, Hamilton S, Albers GW. Collateral effect of Covid-19 on stroke evaluation in the United States. N Engl J Med. 2020;383(4):400-401.

26. Hajdu SD, Pittet V, Puccinelli F, et al. Acute stroke management during the COVID-19 pandemic: does confinement impact eligibility for endovascular therapy? Stroke. 2020; 51(8):2593-2596.

27. Sweid A, Jabbour P, Tjoumakaris S. Letter to the editor: Incidence of acute ischemic stroke and rate of mechanical thrombectomy during the COVID-19 pandemic in a large tertiary care telemedicine network. World Neurosurg. 2020; 140:491-492.

28. Antony J, James WT, Neriamparambil AJ, et al. An Australian response to the COVID-19 pandemic and its implications on the practice of neurosurgery. World Neurosurg. 2020;139: e864-e871.
29. D'Amico RS, Baum G, Serulle Y, et al. A roadmap to reopening a neurosurgical practice in the age of COVID-19. World Neurosurg. 2020;139:289-293.

\section{Disclosures}

The authors report no conflict of interest concerning the materials or methods used in this study or the findings specified in this paper.

\section{Author Contributions}

Conception and design: Raneri, Rustemi, L Volpin. Acquisition of data: Raneri, Zambon, Del Moro, Magrini, Ceccaroni, Basso, F Volpin, Cappelletti, Lardani, Ferraresi, Guida, Chioffi, Pinna, Canova, d'Avella, Sala. Analysis and interpretation of data: Raneri, Rustemi, Del Moro, Magrini, Ceccaroni, Basso, F Volpin, Cappelletti, Lardani, Ferraresi, Guida, Chioffi, Pinna, Canova, d'Avella, Sala, L Volpin. Drafting the article: Raneri, Rustemi, L Volpin. Critically revising the article: all authors. Reviewed submitted version of manuscript: all authors. Approved the final version of the manuscript on behalf of all authors: Raneri. Statistical analysis: Raneri. Administrative/technical/material support: Raneri, Rustemi. Study supervision: Raneri, Rustemi, L Volpin.

\section{Correspondence}

Fabio Raneri: Ospedale San Botolo, Vicenza, Italy. fabran@ gmail.com. 